\title{
SAFIRE-A: Spectroscopy of the Atmosphere Using Far-Infrared Emission/Airborne
}

\author{
Bruno Carli,* Peter A. R. Ade, + Ugo Cortesi,* Paul Dickinson,\# Michele Epifani,* \\ Fred C. Gannaway, + Alessandro Gignoli,* Corneli Keim, @ Clare Lee, + Claude Meny, \& \\ Jean Leotin, \& Francesco Mencaraglia,* Alexander G. Murray, ${ }^{+}$ \\ IRA G. NOLT, ${ }^{*} *$ AND MARCO RIDOLFI* \\ *IROE-CNR, Florence, Italy \\ ${ }^{+} Q M W$, London, United Kingdom \\ \# IMK-FZK, Karlsruhe, Germany \\ ${ }^{@}$ RAL, Chilton Didcot, Oxfordshire, United Kingdom \\ \&SNCMP, Complexe Scientifique de Rangueil, Toulouse, France \\ **NASA/Langley Research Center, Hampton, Virginia
}

(Manuscript received 5 November 1997, in final form 6 July 1998)

\section{ABSTRACT}

\begin{abstract}
A new instrument named SAFIRE-A (Spectroscopy of the Atmosphere using Far-Infrared Emission/Airborne), which can operate on high-altitude platforms, has been developed for the study of the atmospheric composition through limb-scanning emission measurements. The instrument is a polarizing Fourier transform spectrometer that operates in the far infrared with a resolution of $0.004 \mathrm{~cm}^{-1}$. SAFIRE-A uses efficient photon noise limited detectors and a novel optical configuration, which provide a cold pupil and field stop as well as cold narrow bandpass filters to enhance its sensitivity. The instrument was successfully operated on an M-55 stratospheric research aircraft in the polar regions during the winter 1996-97 Airborne Polar Experiment. The instrument design, aircraft integration, and performances attained in the field campaign are described and discussed. The atmospheric emission spectrum is measured with an rms noise accuracy of $0.5 \mathrm{~K}$ (measured in brightness temperature) in each spectral element near $20 \mathrm{~cm}^{-1}$ with a 30 -s measurement time.
\end{abstract}

\section{Introduction}

In the past five years, much has been learned about the basic mechanisms of ozone loss at high latitudes from aircraft programs such as the European Arctic Stratospheric Ozone Experiment and the Arctic Aircraft Stratospheric Experiment (Proffitt et al. 1993). These programs have produced a good understanding of the perturbed polar chemistry within the polar vortex, but leave many questions unresolved concerning the chemistry and resulting ozone loss outside the vortex, which is affected by vortex processed air.

As part of the continuing effort to characterize ozone chemistry, a new spectroscopic instrument has been specifically designed to make sensitive passive measurements of most of the key species involved in ozone destruction. This instrument measures the far infrared to submillimeter emission of rotational line features of hydrogen, oxygen, and chlorine molecular compounds involved in ozone chemistry. The new instrument,

Corresponding author address: Dr. Bruno Carli, Istituto di Ricerca Sulle Onde Elettromagnetiche, Nello Carrara del Consiglio Nazionale delle Ricerche, IROE-CNR, Via Panciatichi, 64-50127 Firenze, Italy. E-mail: carli@iroe.iroe.fi.cnr.it named SAFIRE-A (Spectroscopy of the Atmosphere using Far-Infrared Emission/Airborne) is herewithin described.

A review of comparable instruments for earth observation measurements from deployable platforms can be found in Kramer (1994). The measurements must be performed above the tropopause in order to avoid water vapor attenuation. Observation from high altitudes permits a sequence of angles above and below the horizon (limb scanning) to be viewed. Retrievals of the vertical concentration of target species can therefore be observed from the tropopause (which in the polar regions can be at less than $10-\mathrm{km}$ altitude) to the flight altitude (about $20 \mathrm{~km}$ for aircraft altitudes and $40 \mathrm{~km}$ for balloon altitudes). Measurements of total column content can be recorded above the flight altitude. Several constituents have been measured in this spectral region with Fourier transform spectrometry (FTS) (see references in Carli and Carlotti 1992), heterodyne (e.g., De Welk et al. 1997), and Fabry-Perot spectrometry (e.g., Pickett and Peterson 1996) instruments. FTS and heterodyne techniques have competing performances at low frequencies (submillimeter region), while FTS and Fabry-Perot techniques have competing performances for single species measurements. Nevertheless, only FTS provides 
broadband multispecies measurements. A high-resolution, balloonborne FTS instrument (Carli et al. 1984) has been operated for many years, but the location of the balloon flights was limited to midlatitudes near the launch facility and the size of the instrument precluded its use in an aircraft, as well as in a polar balloon. Its design also prohibited the exploitation of the current superior photon noise limited detectors. SAFIRE-A is a new instrument that capitalizes on the experience of 10 flights with the balloon instrument, on the new detector technology, and on the design of a space instrument that was studied for the Earth Observing System program. SAFIRE-A was optimized for operation on board the M-55 Geophysica stratospheric aircraft and was used in the Airborne Polar Experiment (APE) during the winter 1996-97 arctic campaign in Rovaniemi, Finland.

The role of SAFIRE-A in this campaign has been to determine the extent of the chemical modifications to the stratospheric air at specific geographical locations [e.g., in the presence of polar stratospheric clouds (PSCs) where heterogeneous chemistry is occurring]. Far-infrared observations have the considerable advantage that they are not affected by the presence of aerosols and PSC particles. Both the scattering and thermal emission from aerosols and PSC particles have negligible effect on the instrument response, since the aerosols have a size much smaller than the wavelengths observed. The target species are selected by the narrowband filters placed in front of two detectors that simultaneously record the output of the FTS. The instrument covers the spectral region from 10 to $250 \mathrm{~cm}^{-1}$, with an unapodized spectral resolution of $0.004 \mathrm{~cm}^{-1}$.

SAFIRE-A has been designed to be a relatively compact and rugged instrument, making it suitable for deployment in geographically important locations (e.g., Tropics, midlatitudes, or polar latitudes) from balloon or aircraft platforms. The main features of the instrument are

- folded optics in the interferometer (factor of 8) to provide tilt compensation and reduce instrument size,

- cold pupils and field stops in conjunction with narrowband filters tuned around selected atmospheric features for optimum detector sensitivity, and

- integration with the high-altitude M-55 Geophysica aircraft for frequent and flexible flights as part of an integrated payload of scientific instruments.

The design, tests, and qualifications of the instrument, as well as the preliminary results obtained in APE during the winter 1996-97 in Rovaniemi, Finland, are described in the following sections.

\section{Instrument layout and optical design}

The instrument is a high spectral resolution Fourier transform spectrometer of the Martin-Puplett type (Martin 1969) with a polarizing beamsplitter. The overall optical layout of the instrument is presented in Fig. 1, which shows the beam propagation from the front end optics (FEO), through the Fourier transform interferometer (FTI), to the cold optics and detector module (CODM). The atmosphere is viewed through a 200- by 40-mm open port located on the right side of the aircraft with respect to the flight direction. The atmospheric signal is collected by a flat limb-scanning mirror $M$ that corrects for the aircraft roll and performs the limb-scan sequence. The scanning mirror is mounted at $45^{\circ}$ to the optical axis of the instrument beam and is rotated about this axis so that the instantaneous field of view (IFOV) is scanned vertically in a plane perpendicular to the aircraft flight direction (see Fig. 2).

An input telescope consisting of two confocal offaxis paraboloids, M2 and M3, provides a 4/9 reduction in the solid angle of the interferometer relative to the solid angle of the atmospheric beam. A 10-mm-diameter intermediate field stop located at the focal point of the telescope minimizes the thermal and turbulent exchange of atmospheric air between the instrument and the external environment.

The Martin-Puplett interferometer is characterized by the use of an input and output polarizer as well as the polarizing beamsplitter. The input polarizer of the FTI, mounted beyond the focal point of the telescope, transmits the component of atmospheric radiation, which is linearly polarized in the vertical plane (see Fig. 2). The orthogonal linear polarization radiation that is reflected by this polarizer originates from a calibration source and provides the second input port of the instrument. By rotating this input polarizer about an axis perpendicular to the optical axis of the beam, a second blackbody source at a different temperature can be observed providing data for in-flight radiometric calibration (see section $3 \mathrm{~b}$ ). The second off-axis paraboloid of the input telescope provides a collimated beam in which the sources at the two input ports (atmosphere and calibration blackbody) are superimposed with orthogonal polarizations.

The collimated beam enters the FTI through the polarizing beam splitter M4, which has its principal axis oriented at $45^{\circ}$ with respect to the directions of polarization of the two sources. Therefore each source is split into two polarized components (parallel and perpendicular to the principal axis of the beamsplitter) of equal amplitude. The parallel vector is transmitted to the moving fold mirrors (MMA) and the perpendicular vector is reflected and folded by the flat mirror M5 toward the moving fold mirrors MMB. Fixed roof top mirrors in each arm of the interferometer reflect the beams back (see inset in Fig. 1) and introduce a folding of the wavefront around the edge of the roof. The dihedral edge of each fixed roof is vertical so that, for a wave polarized at $45^{\circ}$, the folding of the wavefront is equivalent to a rotation of the plane of polarization by $90^{\circ}$. When the polarized components recombine at the beamsplitter, after traveling different optical paths, the one that was 


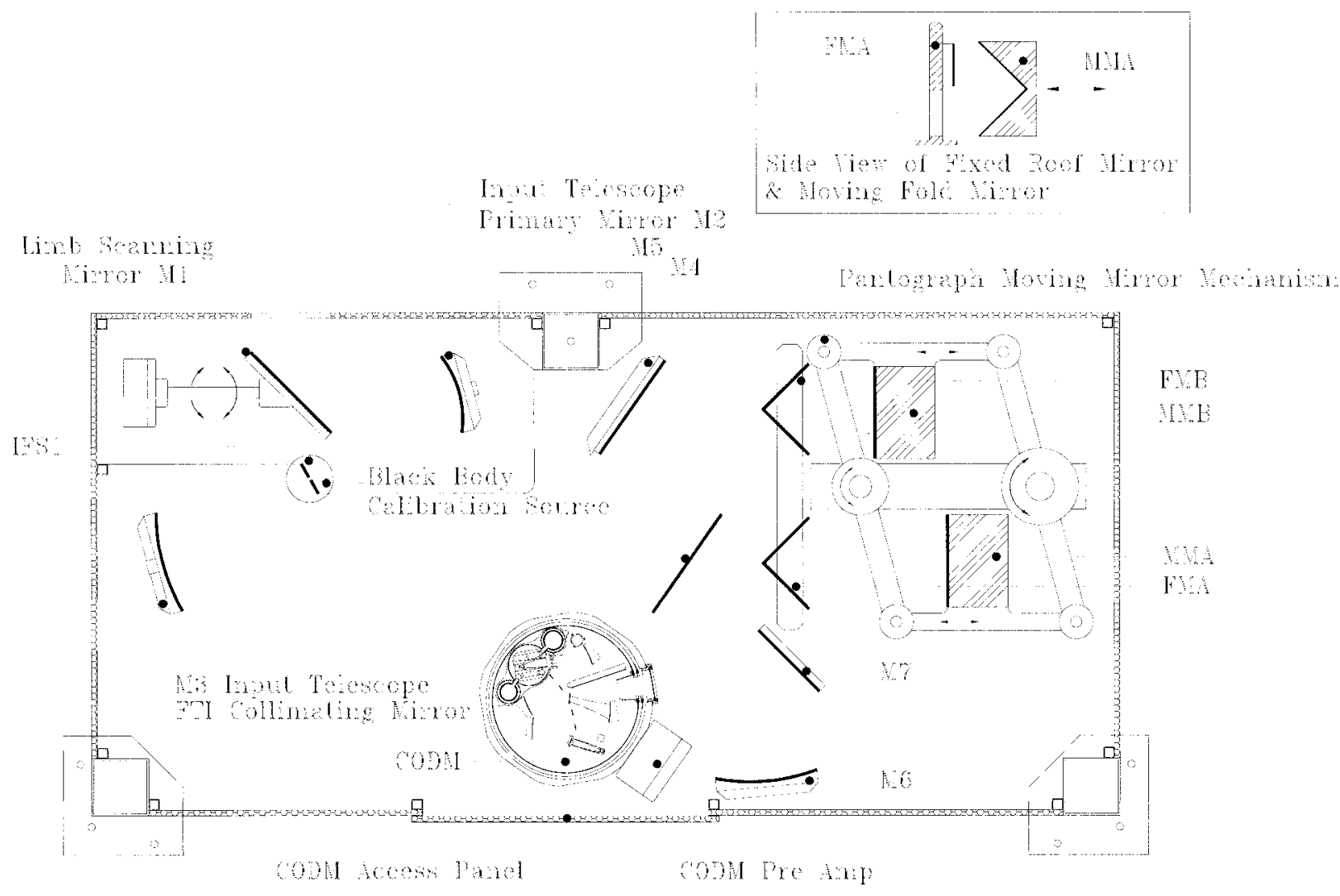

FIG. 1. SAFIRE-A optical layout.

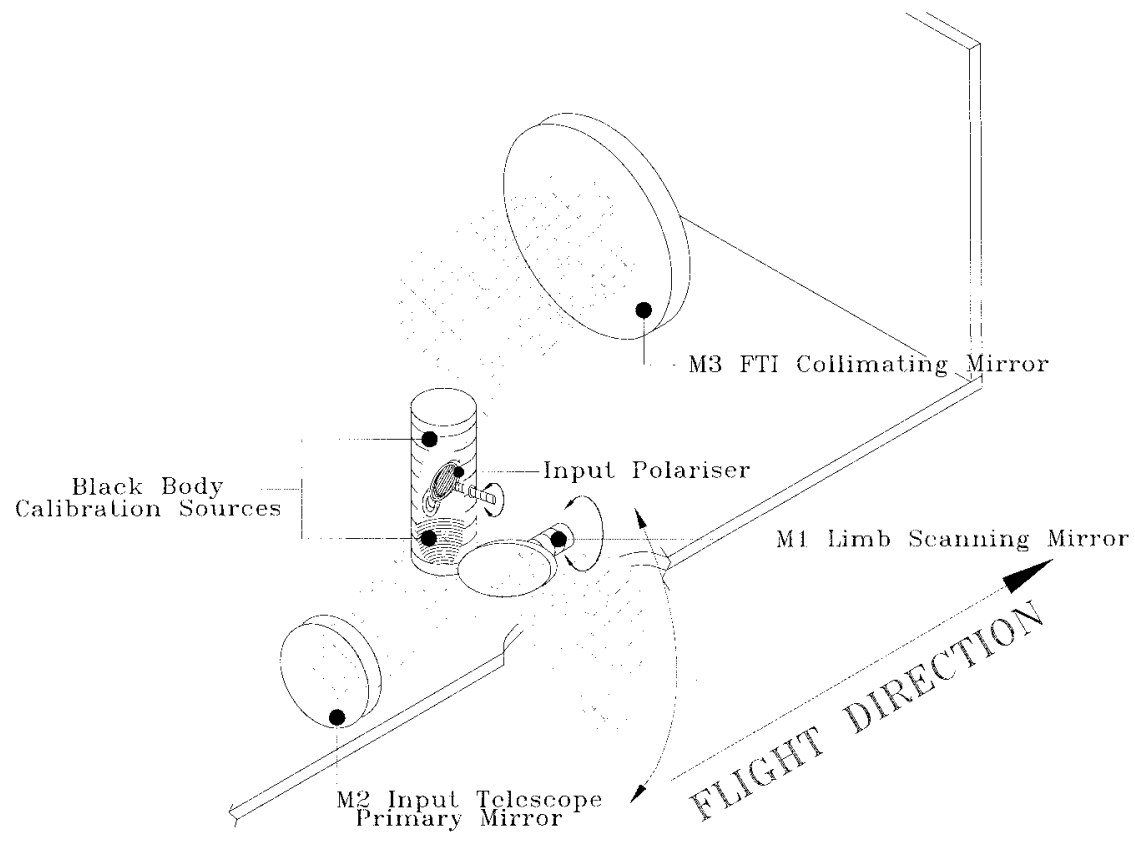

FIG. 2. Limb-scan mirror and calibration unit. 


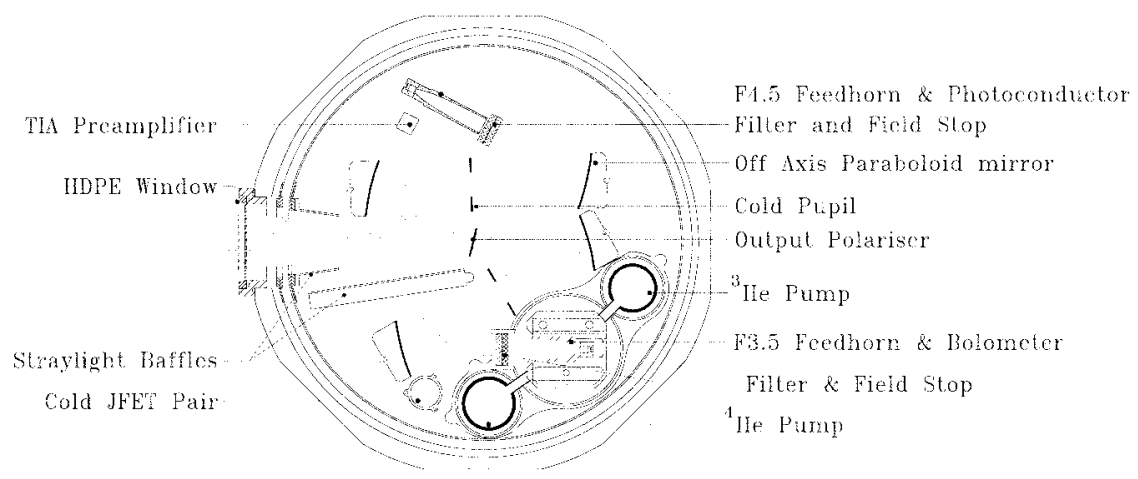

FIG. 3. Cold optics and detector module (CODM).

transmitted is now reflected and the one that was reflected is now transmitted. For an ideal polarizing beamsplitter in a perfectly aligned instrument, the full input signal is transmitted to the output side.

The collimated output beam of the interferometer is condensed into the CODM by an off-axis paraboloid M6 and a folding flat mirror M7. Figure 3 shows the optical configuration of the CODM. The FTI output polarizer (analyzer) is located at the focal point of M6 and creates two output channels (reflected and transmitted beam) in which the components that travelled different paths have now the same polarization and can interfere to produce interferograms. At both output ports we observe the interferogram of the spectral difference between the two input ports. The two interferograms have opposite phase (at zero path difference one interferogram has a positive signal, the other has a negative signal), and both in principle give access to the full spectral range $\left(10-250 \mathrm{~cm}^{-1}\right)$.

Two small condensing mirrors in each channel reimage the focal plane of the paraboloid onto the detector horn apertures (field stops), which are sized to provide the appropriate beam aperture on the sky when projected

TABLE 1. Instrument specifications.

\begin{tabular}{|c|c|}
\hline Type of instrument & $\begin{array}{l}\text { Polarizing Fourier transform interfer- } \\
\text { ometer }\end{array}$ \\
\hline Size & $1800 \times 880 \times 650 \mathrm{~mm}$ \\
\hline Weight & about $400 \mathrm{~kg}$ \\
\hline Measurement technique & $\begin{array}{l}\text { Limb sounding with vertical profile } \\
\text { retrieval from tropopause to flight } \\
\text { altitude }\end{array}$ \\
\hline Measurement time & 30-60 s/interferogram \\
\hline Number of detectors & 2 \\
\hline $\begin{array}{l}\text { Spectral region of detec- } \\
\text { tors }\end{array}$ & $10-250 \mathrm{~cm}^{-1}$ \\
\hline Spectral resolution & $0.004 \mathrm{~cm}^{-1}$ \\
\hline Detector bandwidth & $1-20 \mathrm{~cm}^{-1}$ \\
\hline Vertical resolution & $3 \mathrm{~km}$ \\
\hline Limb step & $1.5 \mathrm{~km}$ in tangent altitude \\
\hline Spectral S/N & $>500: 1$ \\
\hline Throughput & $0.028 \mathrm{~cm}^{2} \mathrm{sr}$ \\
\hline IFOV & $1.4^{\circ}$ \\
\hline
\end{tabular}

back through the optical system. A detector field stop of $10-\mathrm{mm}$ diameter corresponds to an IFOV of $1.4^{\circ}$. A cold pupil is located in each of the parallel beam sections between the two small condensing mirrors. The size and position of the cold pupil defines the size and position of its image in the FTI and is used to limit the beam growth due to its solid angle at the middle position of the interferometer path. A 16-mm-diameter cold pupil corresponds to a 12.8 -cm-diameter beam section in the interferometer.

The small diameter polarizers, made photolitographically, composed of $1-\mu \mathrm{m}$-wide copper strips supported on a thin $(1.5 \mu \mathrm{m})$ mylar membrane with $2-\mu \mathrm{m}$ spacing. For the beam divider a freestanding wire grid polarizer with $10-\mu \mathrm{m}$-thick wires and $25-\mu \mathrm{m}$ spacing or a larger photolithographic beamdivider can be used.

The maximum throughput of the instrument is equal to $0.028 \mathrm{~cm}^{2} \mathrm{sr}$, which allows for resolving powers up to 30000 . The throughput reduction that would be necessary for either higher resolving power or smaller IFOV can be obtained by reducing the detector field stop. A cold narrowband filter is located in front of each detector, to select the atmospheric lines of interest, which together with the cold pupil and field stop is used to limit the radiation flux on the detector and hence the associated photon noise. By using different filters at the two detectors different spectral regions can be simultaneously observed. A summary of the instrument parameters is given in Table 1 and details on the detectors are given in section $3 \mathrm{e}$.

\section{Description of instrument subsystems}

Apart from the basic FTI optics, the instrument includes the following subsystems as shown in the layout in Fig. 4:

- limb-scanning mirror (LSM);

- attitude and heading reference system (AHRS);

- instrument calibration unit (ICU);

- scanning mechanism of the FTI;

- He-Ne laser interferometer;

- cryostat and cold optics and detectors module; and 


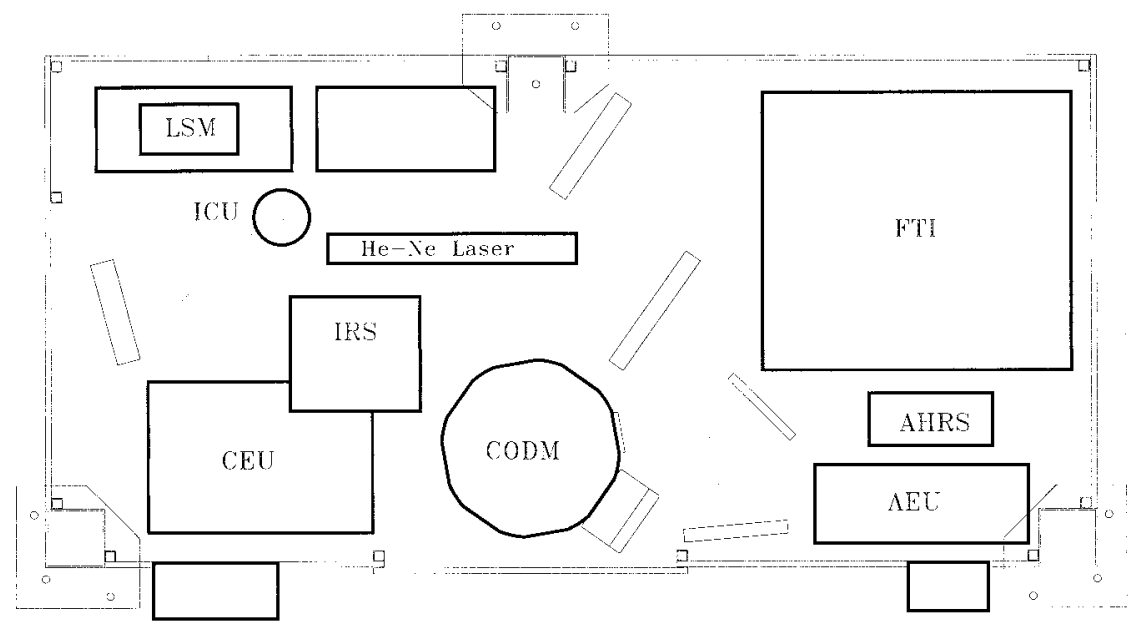

FIG. 4. Block diagram showing SAFIRE-A subsystems.

- flight electronics and data recording central electronics unit (CEU) analogue electronics unit (AEU), and instrument recording system (IRS).

A description of each of these relevant subsystems is given in the following sections.

\section{a. Limb scanning mirror}

The control system of the limb-scanning mirror in the FEO must be able to accurately set the limb view angle and correct for possible changes of the attitude of the platform. In the case of a balloon platform changes in the attitude are rather slow, but a high pointing accuracy must be attained (typically about 1 arcmin, to maintain a tangent height accuracy of $100 \mathrm{~m}$ for a tangent altitude view to the $30-\mathrm{km}$ level below the flight altitude). In the case of an aircraft platform, the pointing system must respond to more rapid variations, but the pointing accuracy is somewhat less stringent (typically $2-3$ arcmin) because of the lower altitude of the platform. The SAFIRE-A pointing system has been designed for use in both operational situations and uses an externally pro-

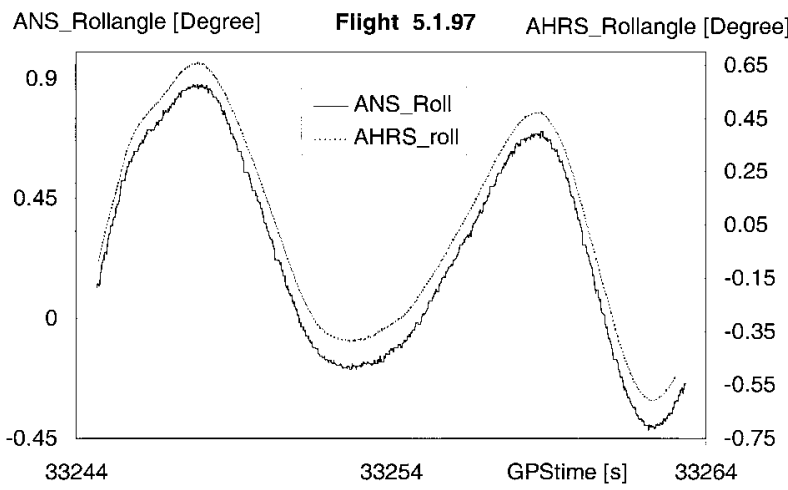

FIG. 5. Comparison between the roll angle measured by the ANS and the AHRS mounted on the SAFIRE-A base plate. vided attitude signal. When used on an aircraft the attitude signal can be provided by the Aircraft Navigation System (ANS). However, there is an uncertainty associated with this control loop since SAFIRE-A is supported on antivibration mounts, which allow some flexure between the roll of the aircraft and the instrument.

During the Rovaniemi campaign, comparative measurements were made between the ANS output and data from an AHRS mounted on the SAFIRE-A base plate. The AHRS is an attitude system developed for another aircraft remote sensing instrument, the Michelson Interferometer for Passive Atmospheric Sounding (MIPAS) (Piesch 1996). The MIPAS AHRS (Seefeldner and Keim 1995) consists of two parts: an inertial navigation system (a combination of gyros and accelerometers) to detect rapid changes of attitude and a Global Positioning System (GPS) to prevent drift errors due to changes in position. The local horizon, which is the reference for the attitude angles, is defined from the geographical position using the WGS-84 Earth model (Decker 1984). With the aid of the GPS, the nominal $3 \sigma$ accuracy of the AHRS is 1.5 arcmin for the two attitude angles (roll and pitch). This high accuracy was confirmed in several balloon flights where the absolute pointing accuracy was determined by a star reference system (Maucher 1995).

The comparison data from the Rovaniemi campaign showed that both systems (ANS and AHRS) observe about the same roll angle, apart from a small constant offset between their mean values throughout a flight (see Fig. 5). When the static offset is removed, the rms of the difference of the two readings is less than 1 arcmin. This shows that the antivibration mounts introduce a negligible instability. A closer study of the ANS data shows that at high frequencies it has a higher fluctuation level than the AHRS, with peak amplitude of about 1 arcmin (Fig. 6). Explanations for this could be either that the ANS detects real vibrations of the M-55 airframe, which are damped by the SAFIRE-A mounts, or 


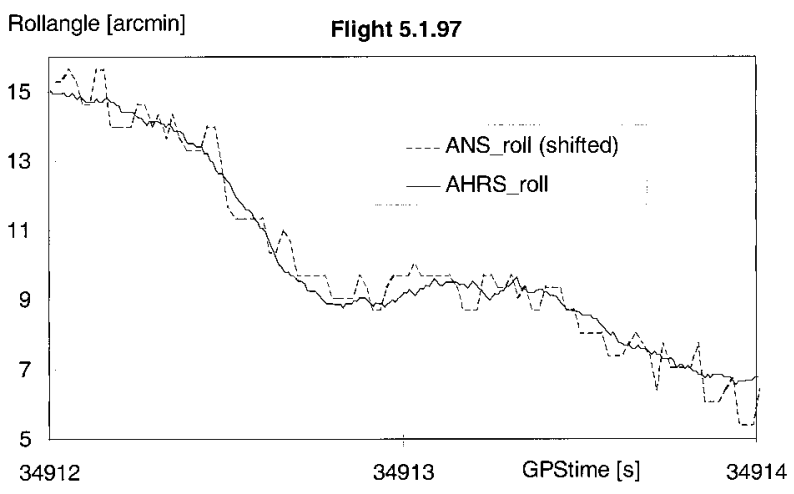

FIG. 6. High-frequency response of ANS and AHRS roll signals.

that the ANS has a measurement noise with a bandwidth larger than the AHRS.

We also have clear evidence of occasional, but significant, discrepancies between the two systems. It is believed that some of these result from different elongations of the left and right antivibration mounts during major aircraft maneuvers. However, although measurable, the differences between the roll angle of the instrument and the roll angle provided by the ANS were either small or of limited duration. The degradation to the recorded data was small so these results validate the operational use of the ANS for roll correction. Naturally, the use of an instrument-mounted AHRS would be better.

\section{b. Instrument calibration unit}

The Martin-Puplett interferometer measures the spectrum of the difference between the signals transmitted and reflected by the input polarizer. The transmitted signal is the atmospheric emission and is varied by changing the pointing of the limb scan mirror, while the reflected signal can be changed by switching the orientation of the input polarizer. By switching the reflected input beam between two reference blackbodies at different temperatures, it is therefore possible to calibrate the instrument response. The blackbodies are made from a conical cavity bored into an aluminium cylinder, which is then blackened with a 1-mm-thick deposition of carbon impregnated Epotek 902 epoxy. This coating provides a reflectivity of less than $13 \%$ over the spectral range from 20 to $650 \mathrm{~cm}^{-1}$. Since at least two reflections occur in the cavity, the blackbodies have an emissivity better than $98.3 \%$. One of the two reference sources is heated to about $100^{\circ} \mathrm{C}$ ("hot blackbody") and its temperature is stabilized by a temperature controller; the other source is kept at the ambient temperature near $0^{\circ} \mathrm{C}$ ("cold blackbody"), and its temperature is passively monitored. By recording two consecutive limb emission measurements at the same pointing angle (usually at a high limb angle of $+10^{\circ}$ ) against first the hot and then the cold blackbody, a calibration is achieved. The difference between the two measured spectra is the difference between the two blackbodies of known radiance.
The instrument and detector responsivity were shown to be constant over each set of measurements, so it was only necessary to record calibration measurements every 15 minutes. An averaged responsivity was then determined for each flight. In this way the accuracy lost through the small differential temperature between the two blackbodies is regained with the averaging process.

\section{c. Scanning mechanism of the FTI}

The two folds that are present in the optical path of the interferometer provide an optical configuration, which is fully compensated for tilt of the moving mirror and is compensated in one direction for lateral shift (Carli 1989). The scanning of the moving mirrors is implemented with a compact pantograph mechanism that moves the mirrors simultaneously in both arms of the interferometer so that as the path to MMA increases, the path to MMB decreases, and vice versa (see Fig. 7). The combination of the mechanical doubling of the effective optical path together with the beam folding provides a long optical path difference $(125 \mathrm{~cm})$, for a small mirror stroke $(15.6 \mathrm{~cm})$ in a compact instrument. Furthermore, a torque motor on a central axis of the pantograph can efficiently perform the momentum compensated motion (Barbis et al. 1994; Brotini et al. 1993). A constant mechanical offset is present in one arm of the interferometer to allow single-sided interferograms to be recorded over the maximum path difference available and thus decreasing the spectral resolution by nearly a factor of 2 .

The motion of the torque motor is controlled using a resolver mounted on the second central axis of the pantograph in a servo loop. A digital control of the angular speed provides a constant linear mirror speed. Interferograms are acquired both in forward and reverse motion.

Figure 8 shows the speed distribution, as measured by laser fringe time intervals with the laser interferometer (see next section) during an interferometric sweep performed in a quiescent condition on the ground [narrow distribution (A)]. For comparison an interferometric sweep performed in perturbed flight conditions is shown in the broader distribution (B). From a Gaussian fit of the two distributions we obtain an rms speed error of $\pm 2 \%$ on the ground and of $\pm 5 \%$ in flight conditions. As will be discussed in section $3 \mathrm{f}$, the speed error observed in flight conditions causes a sampling error of negligible amplitude.

\section{d. Laser interferometer}

A laser interferometer is used to determine the regular spatial sampling of the optical path difference. Figure 9 shows the optical arrangement of the laser interferometer, which is a four-channel homodyne system, using polarizing optics. The performances of a laser interferometer in this configuration are discussed in Barbis et al. (1998). The reference source is a frequency stabilized 


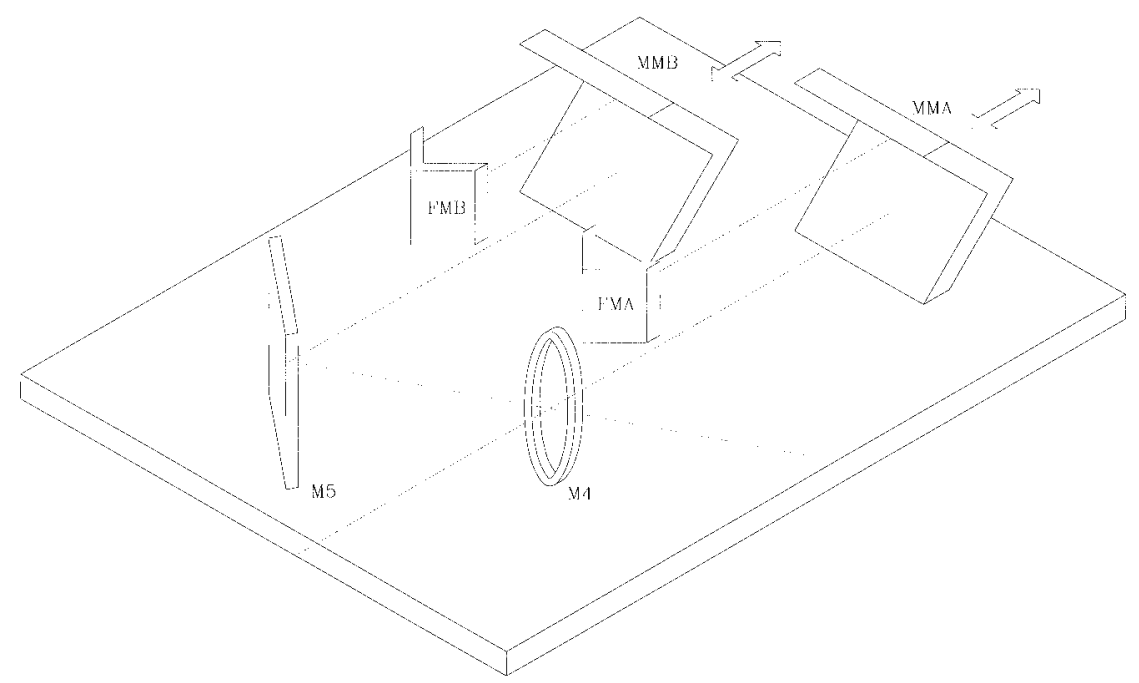

FIG. 7. FTI optical configuration showing double folding of the optical path.

He-Ne 632.8-nm laser (model 117-A by Spectra Physics) with a frequency stability of $1: 10^{8}\left( \pm 2.0 \mathrm{MHz} \mathrm{h}^{-1}\right)$. The optical path of the laser beam is parallel to the farinfrared collimated beams and uses optical components that are rigidly connected to the far-infrared components but optimized for operation in the visible region.

The amplitude beamsplitter (BS) splits the vertically polarized laser beam into both arms of the interferometer. Subsequently, all the reflections encountered in the interferometer involve planes of incidence, which are either parallel or perpendicular to the direction of polarization of the beam so that no change is introduced in the state of polarization. The fixed rooftop mirrors introduce lateral offsets in the returning beams so that they are separated from the input. Before recombination at the beamsplitter, one beam passes through the halfwave plate (HWP), which rotates its plane of polarization by $90^{\circ}$. The beamsplitter produces two outputs in which the two beams that traveled in the two arms

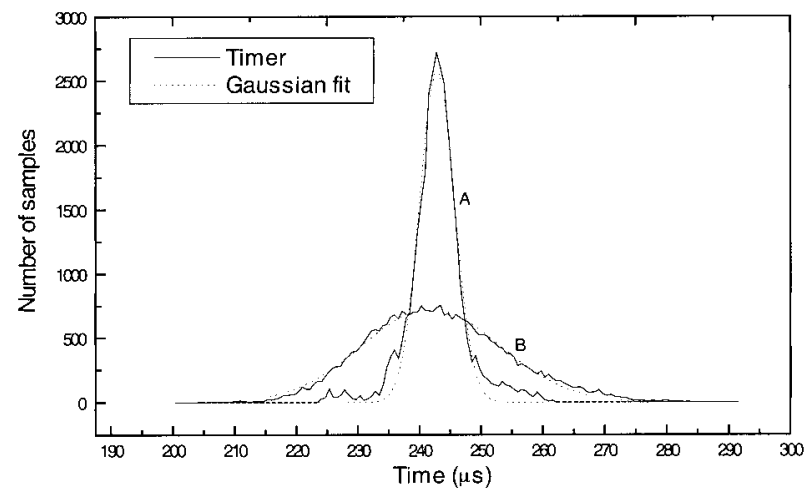

FIG. 8. Scanning speed error distribution in the case of a quiet laboratory environment [narrow peaked distribution (A)] and in flight conditions (B). In both cases a Gaussian curve has been fitted to the distribution (dotted lines). of the interferometer are superimposed but polarized in orthogonal directions. In one output of the interferometer, a quarter-wave plate (QWP) that has its fast axis parallel to one of the polarizations introduces a further phase delay of $90^{\circ}$ between the two planes of polarization, which is equivalent to an additional path difference delay between the two beams. The polarizers $\mathrm{P} 1$ and $\mathrm{P} 2$, whose axes are orientated at $45^{\circ}$ to the two orthogonal polarized beams, further divide the two outputs of the beamsplitter. At P1 and P2, the components of these beams resolved along the polarizer $45^{\circ}$ direction are transmitted and those resolved perpendicular to the polariser are reflected. As the planes of polarization of the outputs from P1 and P2 are necessarily parallel, interference can now take place between the two beams that traveled in the two arms of the interferometer. Four detectors measure the four interference signals. Different interference patterns exist in the four outputs. The reflected and transmitted outputs of each polarizer are in antiphase. Further, there is an additional $90^{\circ}$ path delay between the outputs from P1 and P2 because of the QWP.

The antiphase outputs from P1 or P2 can be combined to eliminate the DC level. The resulting signal from detectors (4-3) provide a measurement of the path difference increment, while the quadrature output from detectors $(2-1)$ provide information on the direction of the motion. The capability of distinguishing the direction of motion makes it possible to have an absolute knowledge of the drive position and so to have a reproducible sampling grid. The laser interferometer provides a measurement of the optical path difference to an accuracy of $10 \mathrm{~nm}$, equal to a fraction of the laser wavelength.

\section{e. Cryostat and cold optics and detector module}

The optical elements of the CODM are cooled to 4 $\mathrm{K}$ in a custom-made cryostat with a cold base plate 


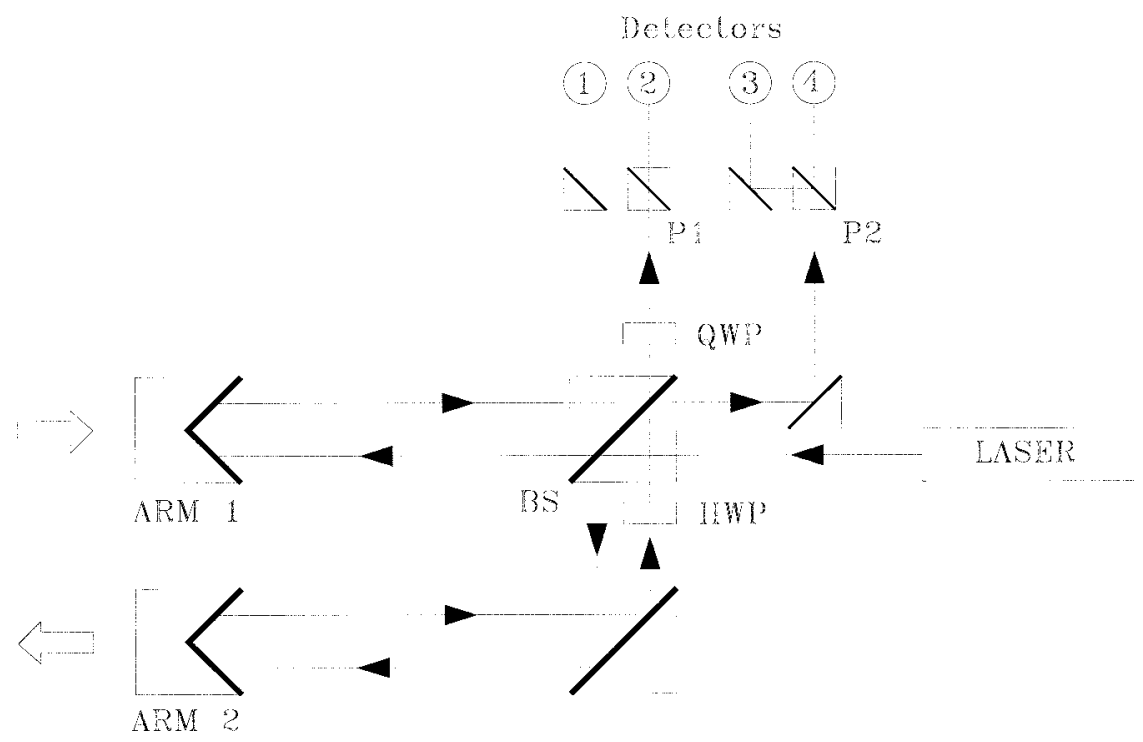

FIG. 9. Optical layout of the laser interferometer used for path difference measurement.

diameter of about $200 \mathrm{~mm}$ for which the optical scheme has been described above (see section 2). A range of photodetectors are available to give good coverage for frequencies between 50 and $500 \mathrm{~cm}^{-1}$ (e.g., stressed Ge: Ga for $50-120 \mathrm{~cm}^{-1}$; Ge:Ga for $120-200 \mathrm{~cm}^{-1}$; Ge:Be for 200-350 $\mathrm{cm}^{-1}$; Si:Ga for 330-500 $\mathrm{cm}^{-1}$ ), while bolometric detectors are necessary for lower frequencies $\left(10-50 \mathrm{~cm}^{-1}\right)$. The temperature requirements for the photoconductive detectors are such that operation from the Helium-4 bath (temperature range 1.6-4.2 K) are ideal, whereas for bolometric detectors much lower temperatures are required (typically $0.3 \mathrm{~K}$ ) for optimum sensitivity.

Narrowband interference filters made with photolithographic metal mesh grids are used in front of each detector and determine the trade-off between band coverage and photon noise. Typically filters with bandpasses from 1 to $20 \mathrm{~cm}^{-1}$ are used. Figures 10 and 11 show the measured transmission of narrowband filters used to select the $29.95 \mathrm{~cm}^{-1} \mathrm{ClO}$ and the $118 \mathrm{~cm}^{-1}$ $\mathrm{OH}$ lines along with the associated metal mesh blocking filters needed to reject FIR to optical power. As indicated in the figure, some of the filters are mounted on the nitrogen and helium radiation shields to reflect the FIR thermal power from the dewar and thus enhance the cryogen hold time as well as effectively blocking highfrequency leaks from the detector environment.

The bolometric detector noise equivalent power (NEP) requirement is that nonphoton noise sources should not contribute significant noise compared to the fundamental photon noise. As we shall see in section 6 , this requires the NEP $\leq 1.0 \times 10^{-15} \mathrm{~W} \mathrm{~Hz}^{-1 / 2}$. Further, its audio speed of response should extend to 200 $\mathrm{Hz}$ to allow signal frequencies to be above any inherently low-frequency noise in the aircraft environment and to allow data to be recorded in a timely way with

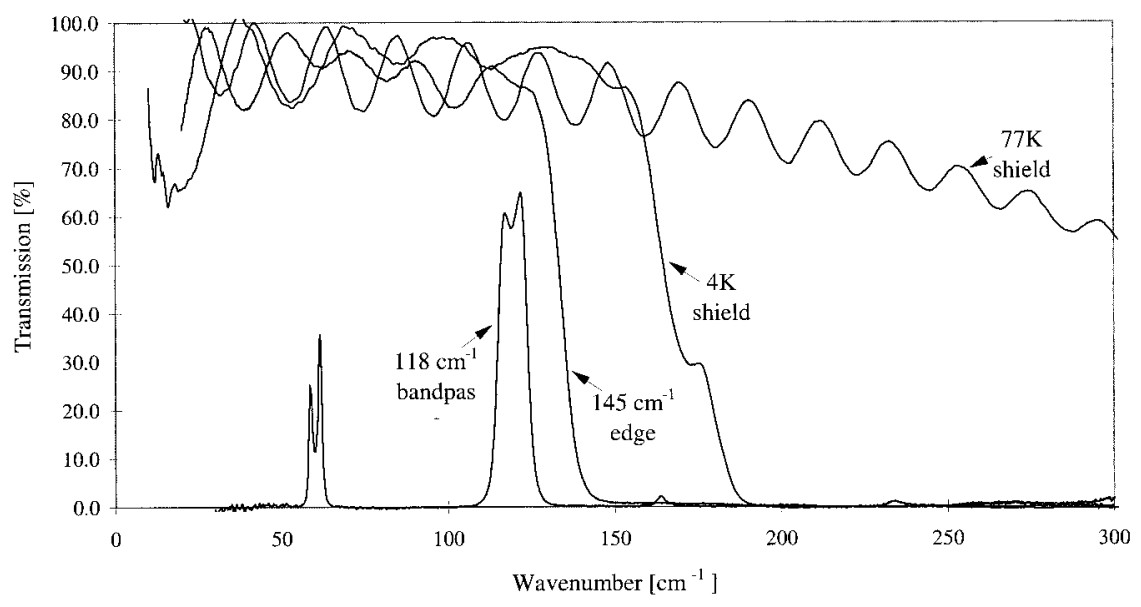

FIG. 10. Spectral transmission of the filters in the photodetector channel. 


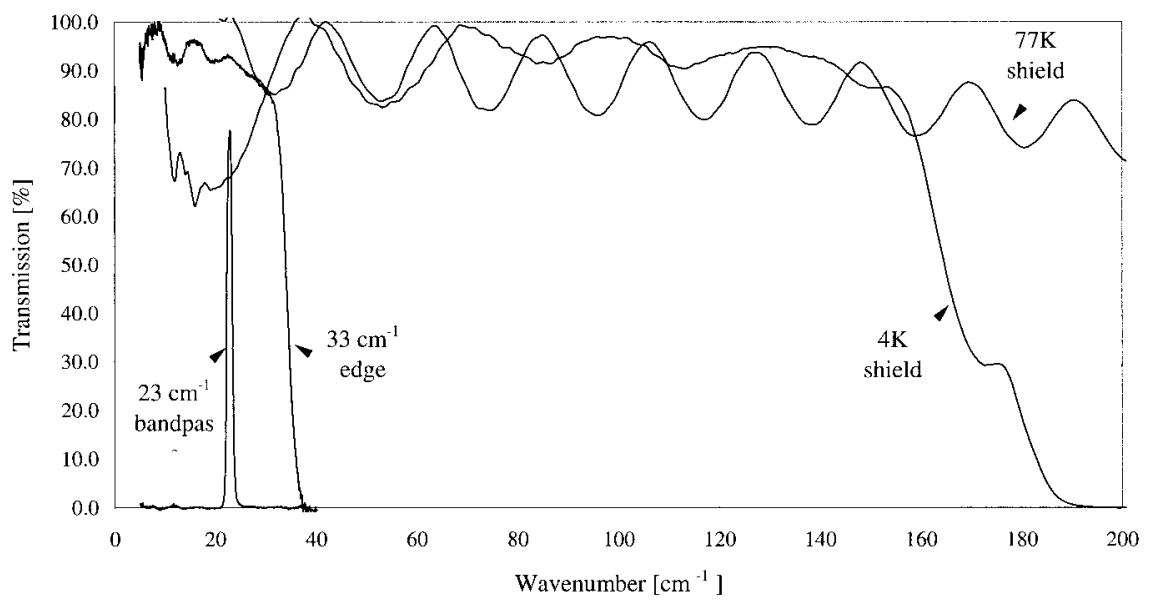

FIG. 11. Spectral transmission of the filters in the bolometer channel.

respect to the aircraft forward motion. The usual tradeoff between sensitivity and speed of response for a bolometric detector dictates that we need to cool it to 0.3 $\mathrm{K}$ to reach these operating parameters. Such a temperature can easily be achieved using Helium-3 adsorption systems. For SAFIRE-A we have developed two CODM systems in two identical cryostats. The first uses two photoconductive detectors for all frequencies above 50 $\mathrm{cm}^{-1}$ and the second utilizes a small Helium-3 stage insert on one channel to enable the use of a bolometric detector simultaneous with a Helium-4 cooled photodetector in the second channel. It is this variant that is shown in Fig. 3. Operationally the cryostats with the different CODMs can be exchanged between flights, enabling a host of species to be observed in the farinfrared.

Laboratory measurements of the bolometer and photoconductor indicated that the required NEP and response bandwidths were met. Operationally the Helium-3 system took $1 \mathrm{~h}$ to cycle after which it stayed at $0.3 \mathrm{~K}$ for over $24 \mathrm{~h}$. The Helium-4 and nitrogen dewars lasted for over $30 \mathrm{~h}$ each, so the cryostat only needed attention once per day and did not interfere with the flight preparations in any way.

\section{f. Flight electronics and data recording}

The power supply is provided by the aircraft but can also be delivered through a dedicated ground link for test operations. Other links with the aircraft include a dedicated analogue link for acquisition of the roll signal from the ANS, housekeeping data on the flight, input on the status of the shutter in front of the window of the instrument, and output on the status of the instrument. The pilot controls the instrument by way of the power supply and the shutter, and verifies through two monitoring lights that normal operations are in progress. No active control is required from the pilot.

A microprocessor in the central electronics unit (CEU) can select the instrument operation (either standby or active) and controls the measurement sequence of the instrument. Instructions prestored on the ground can be implemented either by an internal clock or by the status of the window shutter. For each interferogram the following parameters are chosen: scanning speed of the interferometric sweep, spectral resolution, detector gain, limb scan angle, and the calibration blackbody.

When all the instrument settings are implemented a command is issued to scan the servo drive of the FTI. The drive implements a motion at constant speed between two commanded positions. Constant speed is preceded by an acceleration ramp and is followed by a deceleration ramp. During the constant speed section the interferogram is sampled when the fringe counter passes through each point of the sampling grid. Therefore, the FTI operates in rapid-scan mode with equal space sampling. The scanning speed can be chosen from one of the values indicated in Table 2 so that the spectral bandwidth observed by the detector can be transformed into different electrical bands that are consistent with

TABLE 2. FTI scanning parameters.

\begin{tabular}{ccccr}
\hline \hline & $\begin{array}{c}\text { Electrical/optical } \\
\text { frequency ratio } \\
\left(\mathrm{Hz} \mathrm{cm}^{-1}\right)\end{array}$ & $\begin{array}{c}\text { Nyquist } \\
\text { frequency } \\
\left(\mathrm{cm}^{-1}\right)\end{array}$ & $\begin{array}{c}\text { Number of } \\
\text { interferogram } \\
\text { points }\end{array}$ \\
\hline 0 & 10 & 1 & 2000 & 512000 \\
1 & 20 & 2 & 1000 & 256000 \\
2 & 40 & 4 & 500 & 128000 \\
3 & 80 & 8 & 250 & 64000 \\
\hline
\end{tabular}


the detector speed of response. A constant sampling frequency of about $4 \mathrm{kHz}$ is used with all sampling speeds, so the sampling interval is scaled with the drive speed, which results in a variable Nyquist frequency and a variable number of interferogram points as shown in Table 2.

The first stage amplification is performed by a transimpedance amplifier for the photoconductor and a differential-JFET amplifier for the bolometer, both of which are located inside the cryostat. The second amplification stage with its battery power supply is mounted on the outer cryostat body to minimize radio frequency interference. The preamplified signals are further filtered and amplified by an analogue unit that conditions the signal prior to 16-bit Analog-to-Digital Conversion (ADC). The analog unit exchanges information with the rest of the electronics through fiber optics links and receives electrical power through dcdc converters so that the detectors can be fully isolated from external electrical perturbations. Sampling of the signal by the ADC is commanded by the asynchronous strobe provided by the laser fringe counter after individual time delays have been introduced to compensate for the time constants of the detectors and of the electrical filters. This delay compensation is important because in the case of equal space sampling the sampling error is equal to the speed error multiplied by the uncorrected part of the time delay. In section $3 \mathrm{~d}$ we have seen that in flight condition a $\pm 5 \%$ speed error was experienced with a scanning speed of $5 \mathrm{~mm} \mathrm{~s}^{-1}$ corresponding to an absolute error of $0.25 \mathrm{~mm} \mathrm{~s}^{-1}$. The delay compensation can be selected with a resolution of $10 \mu \mathrm{s}$. In the case of narrowband observations no significant dispersion is expected in the time delay of the electrical signal and the resolution of the delay compensation is the only error in the time domain. The sampling error caused by the mirror drive is therefore equal to $2.5 \mathrm{~nm}$, which is less that the sampling error of $10 \mathrm{~nm}$ from the laser interferometer. The total sampling error is therefore negligible in comparison to the wavelengths $(>50 \mu \mathrm{m})$ at which the instrument operates.

The interferograms and path difference signals, together with aircraft housekeeping data (flight parameters), the instrument housekeeping data (temperature sensors, laser container pressure, etc.), and data on the status of electronic systems and servo mechanisms, are collected in a recording frame and asynchronously stored by an onboard IRS. The recording system is a commercially available aircraft-compatible optical disc recorder with a single side disk capacity of 0.65 Gbytes. To be used in the Geophysica it was sealed in a pressurized box. With a maximum data rate of about 30 kbytes $\mathrm{s}^{-1}$ it allowed continuous operation of SAFIRE-A for more then $6 \mathrm{~h}$ (a typical Geophysica flight was less then $6 \mathrm{~h}$ ).

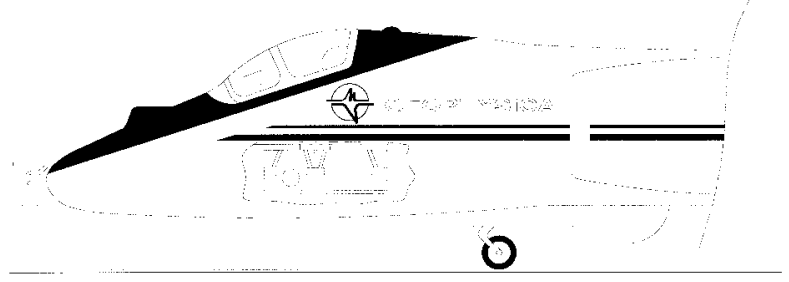

FIG. 12. Mechanical interface of SAFIRE-A with the M-55 Geophysica aircraft.

\section{Instrument structure}

The mechanical structure of the SAFIRE-A spectrometer consists of a single box containing all the instrument subsystems. The base plate of the box provides a rigid optical bench on which the optical components are assembled. This plate is suspended from the body of the aircraft by means of three columns, which are attached to the aircraft via antivibration mounts. The external walls of the box are made of honeycomb panels that limit the thermal and electromagnetic interference (EMI) exchanges of the instrument with its environment, but do not provide any pressure control. The dimensions of the overall system are $880 \times 1800 \times 650 \mathrm{~mm}$ and the total weight is $370 \mathrm{~kg}$ (see Table 1).

The instrument is installed in an unpressurized bay beneath the cockpit by suspension from the aircraft airframe (see Fig. 12). The viewing window is located on the right side of the aircraft with respect to the flight direction. A mechanical shutter in front of the viewing port protects the input optics during ascent and descent and is opened by the pilot when the aircraft reaches the flight altitude.

Access to the instrument for ground operation is possible through a removable hatch on the left side of the aircraft. Replenishment of the cryogenics in the cryostat containing the CODM requires that this hatch is opened after the flight and the cryostat is removed for servicing. Since the cryostat can operate unattended for over 24 $\mathrm{h}$ this activity only has to be performed once per day.

In order to limit the possible cooling of the instrument during the flight, two heaters are mounted on the base plate (actively servoed by temperature sensors) and radiative exchange is reduced by aluminium foil wrapped around the instrument. Mechanical vibration of the instrument is reduced by the antivibration mounts (see section 3a) and acoustic noise due to air turbulence entering the instrument cavity through the viewing port is kept to a minimum by placing a limiting aperture at the focal plane of the input telescope.

\section{Qualification tests}

Ground and flight tests were carried out to qualify the SAFIRE-A spectrometer for flight operation on board the M-55 Geophysica aircraft and to characterize the instrument environment. 
The mechanical structure of the instrument and its interface with the aircraft were tested by means of a flight payload simulator of the spectrometer (mock-up). The SAFIRE-A mock-up is a mass and thermal model of the instrument, having exactly the same weight, dimensions, power dissipation, mechanical structure, and interfaces as the real instrument. The mock-up passed the vibration and shock tests performed at ENEA-Casaccia laboratories in accordance with RTCA/DO-160C standards.

Subsequently the mock-up was installed on the aircraft and used for some flight tests during which the vibration levels at the attachment points and inside the instrument, as well as the temperature at different locations inside the spectrometer box, were measured. These measurements confirmed that the instrument environment is less severe than the environment of the payload bay because of the weak thermal and mechanical linkage.

EMI tests and environmental tests (pressure and temperature) were finally performed on the flight instrument. Both tests were satisfactory after some minor improvements of the filtering at the electrical interfaces.

\section{Instrument performances during the APE Arctic campaign}

During the Rovaniemi Arctic campaign in winter 1996-97 the SAFIRE-A instrument operated in all seven M-55 Geophysica missions. Here we present typical housekeeping data for some of the key environmental parameters recorded during the flights along with sample data, which is used to compare the instrument performance with expectations.

\section{a. Temperature}

Figure 13 shows the instrument temperature during a typical flight. In the figure we have labeled the most significant events (takeoff, shutter open, landing), which would affect the thermal behavior of the instrument environment.

The only significant thermal loss path is via heat conduction through the antivibration mounts. However, the large thermal inertia of the instrument along with its thermal insulation and the electrical heating in both the electronics and some localized thermal control units maintains the instrument temperature above $-5^{\circ} \mathrm{C}$. The upper curve of Fig. 13 shows that the temperature measured near the central beamsplitter (M4) decreased slowly although the external temperature was below $-70^{\circ} \mathrm{C}$ (lower curve in Fig. 13). The intermediate curve of Fig. 13 shows the temperature of the limb scanning mirror compartment, which is most exposed to the external environment to be almost $10^{\circ} \mathrm{C}$ lower than the central instrument temperature. The temperature drop when the shutter opens is clearly visible in the data.

It is therefore evident that there were no severe tem-

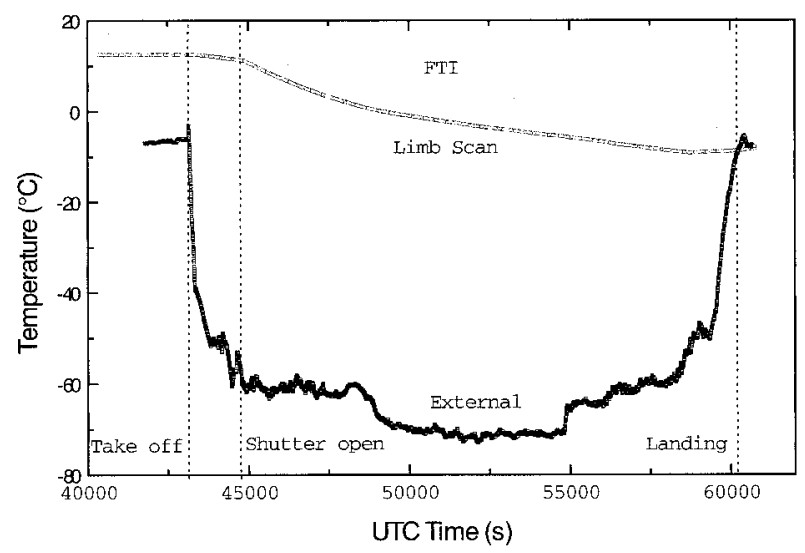

FIG. 13. SAFIRE-A temperature during the Geophysica flight of 31 December 1996. Shown are the external temperature (lower curve), temperature inside the compartment of the limb scanning mirror (middle curve), and inside the interferometer during a typical flight.

perature excursions and indeed the operational range is within the capabilities of most laboratory instrumentation.

\section{b. Vibration levels}

Mechanical vibrations inside the instrument were measured during the engineering flights using accelerometers in the mock-up instrument. No accelerations greater than $0.1 \mathrm{~g} \mathrm{rms}$ were observed at the instrument for the three axes. During the flights with SAFIRE-A no direct measurements of the accelerations were made, but several key systems were monitored for the effects of vibration as follows.

- Significantly, no change was observed in the detector noise from laboratory to flight conditions indicating that the differential amplifiers and cold-stage Kevlar thread supports were effective in eliminating any residual effects.

- As shown previously, in Fig. 8, the accuracy of the mirror drive was degraded in flight by low-frequency oscillations that introduced additional errors into the uncertainty of the mirror position. However, the overall amplitude of the resulting error envelope was acceptable for the effective time-delay compensation and long wavelengths sampled in SAFIRE-A.

- There was no increase in the jitter of the limb-scanning mirror during flight greater than the 20 arcsec sensitivity resulting from the limitation of our angular measurement system.

- A major problem was encountered with the beamsplitter. Two beamsplitters were available for the APE flights: a free standing wire grid polarizer and a photolithographic. Figure 14 shows a comparison of the central portion of interferograms recorded with the two beamsplitters. A good quality interferogram is obtained with the freestanding wire beamsplitter (B), 

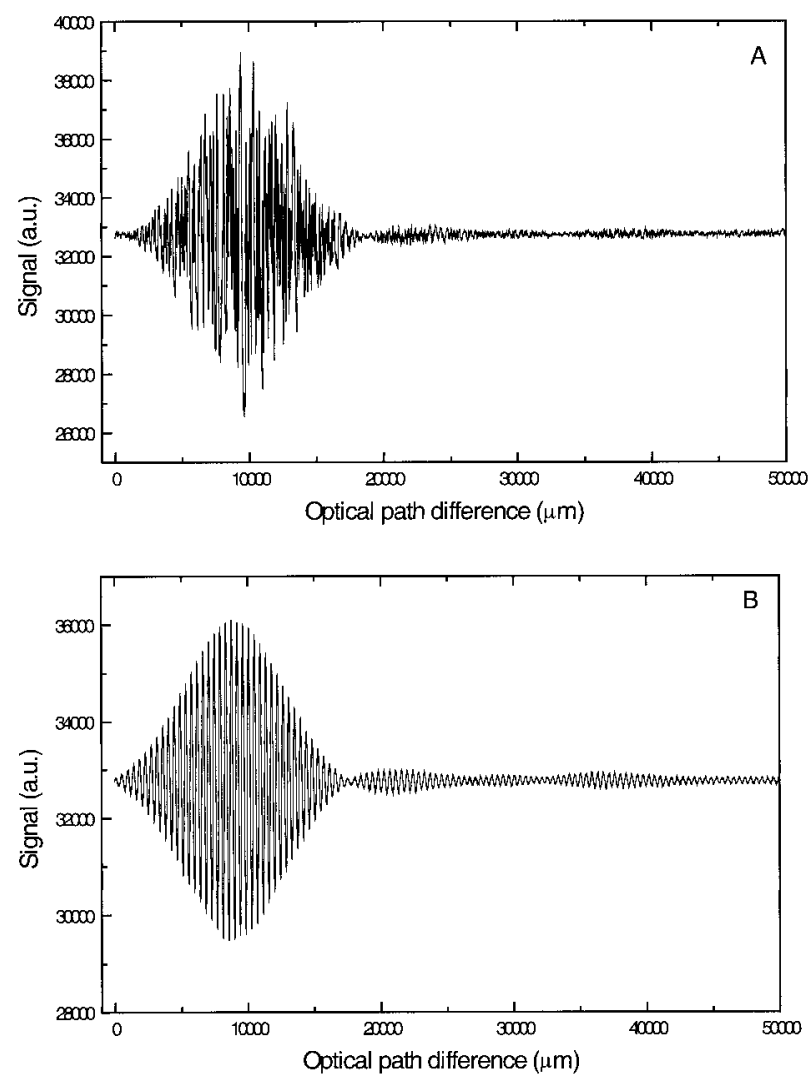

FIG. 14. The central portion of an interferogram taken with a photolithographic polarizer on a substrate (curve A) compared with a free standing wire grid polarizer (curve B).

while large distortions are observed with the photolithographic beam divider (A). The distortions are due to path difference variations introduced by oscillations of the plane of the beamsplitter. The fact that only the substrate-mounted beamsplitter had this problem indicates that the oscillations are caused by acoustic noise rather than mechanical vibrations transmitted through the mount. In order to eliminate this problem we plan to implement better acoustic isolation of the FTI compartment from the limb scanning mirror compartment and thus reduce the transmission of turbulence caused by airflow across the open window aperture in future flights. During the Rovaniemi campaign this problem required the use the free standing wire polarizer, which limited the use of the instrument to the low-frequency channel with the bolometric detector.

\section{c. Spectral resolution}

SAFIRE-A has a theoretical spectral resolution of $0.004 \mathrm{~cm}^{-1}$, defined in terms of the period of independent spectral samples (equal to the inverse of twice the maximum optical path difference). The theoretical instrument line shape (ILS) is a $\sin (x) / x$ function con-

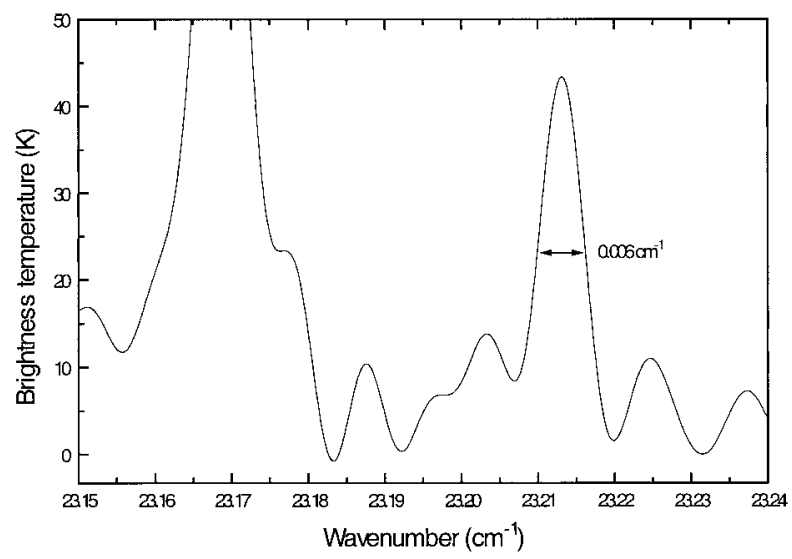

FIG. 15. Full-width half-maximum (FWHM) of a weak narrow atmospheric feature observed in a spectrum recorded with pointing at $10^{\circ}$ above the horizon, which provides an experimental determination of the spectral resolution of the instrument.

volved with a box car function due to the finite solid angle, and it has a full width at half-maximum (FWHM) of about $5 \times 10^{-3} \mathrm{~cm}^{-1}$. The optical design of the CODM (see Fig. 3) makes sure that a minimum angular spread of the beam (compatible with the solid angle) is present in the FTI. Figure 15 shows an isolated atmospheric feature in a spectrum observed at $+10^{\circ}$ above the horizon. The feature is sufficiently narrow and weak to be a good approximation of a Dirac delta function source so the measured line should approximate to the ILS. The measured FWHM of this feature is $6 \times 10^{-3}$ $\mathrm{cm}^{-1}$. Considering that the measured FWHM is also affected by the finite width of the selected feature, the observed linewidth verifies the near theoretical performance in terms of spectral resolution.

\section{d. Instrument stability}

As stated in section $3 \mathrm{~b}$, the calibration process involves the subtraction of two consecutive scans referenced against the cold and hot blackbody sources, respectively. As a check on the calibration reproducibility we have compared in Fig. 16 the calibration scale for scans recorded at a $17-\mathrm{km}$ altitude with scans recorded later at a $20-\mathrm{km}$ altitude, where both the ambient temperature and pressure have changed.

This responsivity curve characterizes the shape and width of the bandpass filter used in front of the detector to limit the photon noise. The good overlap of these plots verifies the good stability of the instrument responsivity as a function of ambient temperature and pressure changes.

\section{e. Signal to noise}

The signal-to-noise ratio $(\mathrm{S} / \mathrm{N})$ of the spectra is defined as the ratio of the signal of the largest atmospheric emission (equal to about the emission of a $210 \mathrm{~K}$ black- 


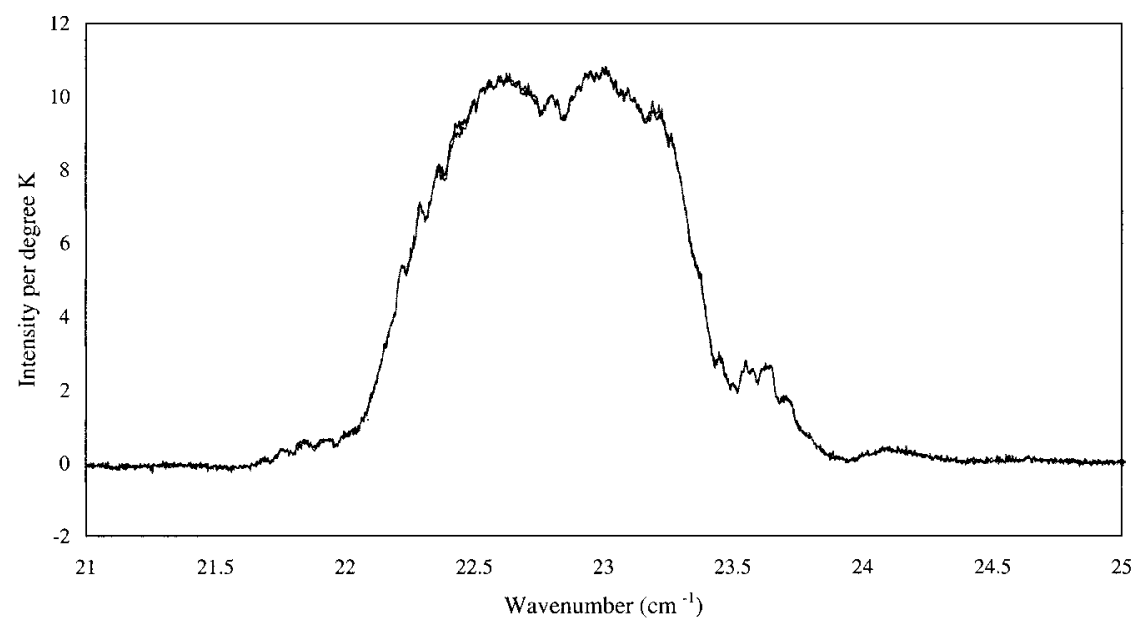

FIG. 16. Comparison of the instrument responsivity at 17 - and 20-km flight altitudes.

body) divided by the rms of the noise measured in regions with no signal. The rms noise is less than $0.5 \mathrm{~K}$ and the $\mathrm{S} / \mathrm{N}$ of the observed spectra is greater than 500:1.

Figure 17 shows the reproducibility of six calibrated spectra measured at the same limb angle (horizontal direction) during a 20 -min period in which the aircraft flew at constant altitude $(20 \mathrm{~km})$. The figure shows both the superpositions of the spectra and their standard deviation. The rms of the variation, shown in an expanded scale in the lower panel, is due to detector noise, calibration errors and variation of the atmospheric source. Larger variations are observed at those frequencies where atmospheric lines are present, confirming that the main source of variation is the source variability rather than measurement error. The measurement error is, however, expected to be the dominant cause of variation at

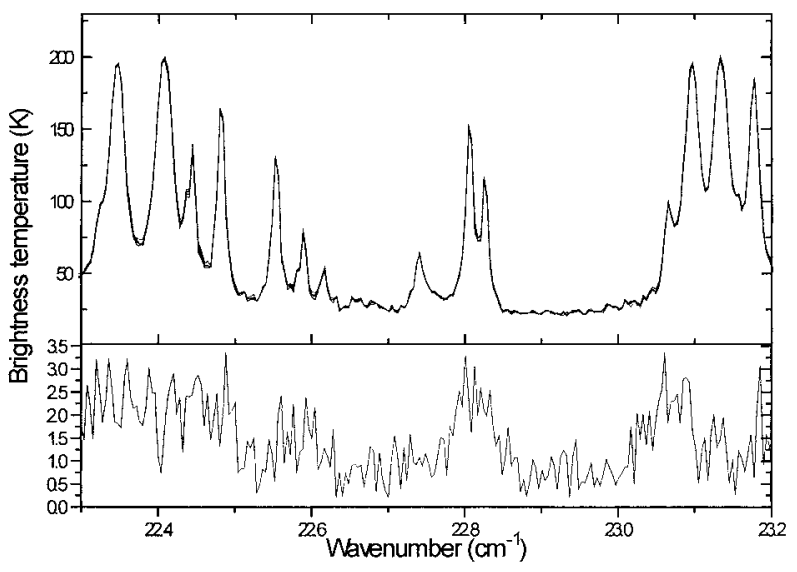

FIG. 17. Reproducibility for a sample of six calibrated spectra of the atmospheric emission measured at the limb angle of $0^{\circ}$ from the altitude of $20 \mathrm{~km}$. The top curve shows the superposition of the six spectra and the bottom curve shows the standard deviation of the statistical distribution of the measurements with a highly expanded ordinate scale. low and high frequencies where the instrument responsivity decreases.

\section{f. Comparison of the signal-to-noise ratio with values expected from a model}

Ideally, the detector requirement is that it should be limited by the photon noise fluctuations arising from the signal power detected in the observation band rather than photon noise from the residual instrument emission. Here we develop a simple spreadsheet model for the noise power sources as seen by the detector.

Basically the detector views the atmosphere in a narrow wavelength band determined by the bandpass filter at the detector. The power incident from frequencies outside of the filter passband is essentially zero, as the filter is at $4 \mathrm{~K}$. The power in the passband from the atmosphere is, however, attenuated by the instrument inefficiencies and, because the instrument is at ambient temperature, it will also emit power to the detector. This instrument emission will degrade the detector performance whether or not it is interferometrically modulated because the incident power will be associated with an increase in the photon noise level. To model the expected $\mathrm{S} / \mathrm{N}$ ratio we estimate the power from the different sources (the atmosphere, the second port, the instrument and the detector), and determine the photon noise resulting from the aggregate power. The $\mathrm{S} / \mathrm{N}$ is the atmospheric signal power $\mathrm{S}$ that is interferometrically modulated divided by the total photon noise.

The radiant power arriving at the detector from a source is given by

$$
P_{\text {SOURCE }}=A \Omega \epsilon_{\text {source }} t_{\text {opt }} B(\bar{\nu}, T) \Delta \bar{\nu},
$$

where $\bar{\nu}$ is the band center in wavenumber, $T$ is the source temperature, $\boldsymbol{\epsilon}_{\text {source }}$ is the emissivity of the source, $t_{\text {opt }}$ is the optical efficiency of the optics located from the source to the detector, $B$ is the Planck function, and $\Delta \bar{\nu}$ and $A \Omega$ are, respectively, the bandwidth and the 
throughput with which the source is observed by the detector. The interferometric modulation of the source flux reduces the detected signal further so that the actual signal detected is given by

$$
P_{\text {Detected }}=\phi_{\text {mod }} A \Omega \epsilon_{\text {source }} t_{\text {opt }} B(\bar{\nu}, T) \Delta \bar{\nu}_{\text {res }},
$$

where $\phi_{\text {mod }}$ is the interferometric modulation factor of the FTI, which includes the optical, interferometric, and electrical efficiencies, and $\Delta \bar{\nu}_{\text {res }}$ is the source spectral bandwidth resolved in one spectral element. The photon noise contribution to the detector NEP arising from the radiant power $P_{\text {SOURCE }}$ incident at the detector depends on the type of detector used. For a photoconductive detector of quantum efficiency $\eta$ the photon shot noise dominates, so it can be estimated from

$$
\mathrm{NEP}_{\text {phot }}=\left(\frac{2 h c \bar{\nu} P_{\text {SOURCE }}}{\eta}\right)^{1 / 2} .
$$

By viewing a modulated blackbody source in the laboratory of known temperature, aperture, and distance, measurements can be made of the detector NEP (bolometer or photoconductor) using the above equations. For the photoconductor it is common practice to express this as a detective quantum efficiency (DQE) through the relation

$$
\mathrm{DQE}=\left(\frac{\mathrm{NEP}_{\text {phot }}}{\mathrm{NEP}_{\text {measureed }}}\right)^{2} .
$$

The DQE for the Ge:Ga photodetector system used here was $8 \%$.

The measured bolometer NEP was $3.6 \times 10^{-15} \mathrm{~W}$ $\mathrm{Hz}^{-1 / 2}$ in line with the photon noise limited estimates, with an inherent NEP of $1.0 \times 10^{-15} \mathrm{~W} \mathrm{~Hz}^{-1 / 2}$ estimated from the electrical characteristics. For a bolometric detector, where photon wave noise must be included, it is given by (Holland et al. 1996)

$$
\mathrm{NEP}_{\text {bol }}=\left[2 P_{\text {SOURCE }}\left(h c \bar{\nu}+\epsilon_{\text {source }} t_{\mathrm{opt}} k T\right)\right]^{1 / 2} .
$$

In the above equations $c$ is the speed of light and $k$ is Boltzmann's constant. To estimate the $\mathrm{S} / \mathrm{N}$ ratio per spectral element achievable from one interferogram, which takes $\tau$ seconds to record, we use

$$
\mathrm{S} / \mathrm{N}=\frac{P_{\text {Detected }} \sqrt{2 \tau}}{\mathrm{NEP}},
$$

where the NEP is appropriate to either the photoconductor or bolometer. The factor of 2 accounts for the $\mathrm{NEP}$ being defined per $\mathrm{Hz}^{1 / 2}$ (equivalent to an integration time of $0.5 \mathrm{~s}$ ).

Using these equations we can calculate the photon noise contributions from each optical element in the beam path taking account of the expected losses in transmission to the detector. This spreadsheet model therefore also determines the overall transmission for both

\begin{tabular}{|c|c|c|}
\hline & Ideal FTI & Realistic FTI \\
\hline $\begin{array}{l}\text { Power at detector fron } \\
\text { atmosphere in } 1 \\
\mathrm{~cm}^{-1} \text { band }\end{array}$ & $1.19 \mathrm{nW}$ & $0.47 \mathrm{nW}$ \\
\hline \multicolumn{3}{|l|}{ NEP contributions } \\
\hline $\begin{array}{l}\text { Source } \\
\text { Second port } \\
\text { Instrument } \\
\text { Detector } \\
\text { Total }\end{array}$ & $\begin{array}{l}2.12 \times 10^{-15} \mathrm{~W} \\
2.71 \times 10^{-15} \mathrm{~W} \\
0 \\
1.00 \times 10^{-15} \mathrm{~W} \\
3.58 \times 10^{-15} \mathrm{~W}\end{array}$ & $\begin{array}{l}0.98 \times 10^{-15} \mathrm{~W} \\
1.22 \times 10^{-15} \mathrm{~W} \\
1.14 \times 10^{-15} \mathrm{~W} \\
1.00 \times 10^{-15} \mathrm{~W} \\
2.18 \times 10^{-15} \mathrm{~W}\end{array}$ \\
\hline $\begin{array}{l}\text { Signal from opaque } \\
210 \mathrm{~K} \text { line per } \\
\text { resolution element }\end{array}$ & $1.19 \times 10^{-12} \mathrm{~W}$ & $3.75 \times 10^{-13} \mathrm{~W}$ \\
\hline $\begin{array}{l}\text { S/N per resolution } \\
\text { element for a } 30-\mathrm{s} \\
\text { interferogram }\end{array}$ & 2567 & 1318 \\
\hline
\end{tabular}
modulated and unmodulated signal components, allowing sensitivity estimates to be made. The sensitivity is calculated in terms of the $\mathrm{S} / \mathrm{N}$ ratio, which can be di-
TABLE 3. FTI sensitivity.

rectly compared with actual flight measurements made with SAFIRE-A. Table 3 gives output data from the spreadsheet for the long wavelength bolometric channel centred at $23 \mathrm{~cm}^{-1}$ with a $1 \mathrm{~cm}^{-1}$ bandpass. We have calculated data for the realistic case of viewing an opaque line at the aircraft flight altitude (blackbody at about $210 \mathrm{~K}$ ) with a reference blackbody at $273 \mathrm{~K}$ in the second port. The throughput for this long wavelength channel is $0.028 \mathrm{~cm}^{2} \mathrm{sr}$ and the FTI resolution is $0.004 \mathrm{~cm}^{-1}$. To appreciate how close this instrument is to fundamental limits we give data for two situations; one assumes an ideal (nonlossy) FTI, the other assumes realistic estimates for all the optical components and is for a realistic FTI.

As expected the major noise sources are the atmosphere itself and the emission from the second port calibration blackbody. The spreadsheet model shows that the instrument losses have an interesting effect: they not only reduce the signal by a factor of about 3.2 , but also reduce the noise contributions by a factor of 1.7 so the overall loss in performance is only a factor of 2 . The noise reduction originates from losses in the CODM only. Reducing the temperature of the nonideal FTI or of the second port source to $4 \mathrm{~K}$ only gives a marginal increase in performance $(\mathrm{S} / \mathrm{N}=1542,1582$, respectively) whereas reducing both the FTI and the second port to $4 \mathrm{~K}$ would give significant gain ( $\mathrm{S} / \mathrm{N} \rightarrow 2022)$. Clearly there is not a strong argument for cooling this type of polarizing, low-emissivity FTI for atmospheric studies.

The observed value of about 500:1 is satisfyingly close to our estimates above but suggests that either we have additional losses that are not accounted for in our model or we have additional noise in the flight data from the M-55 aircraft environment. We will be taking further laboratory calibration data to understand which is most likely. 


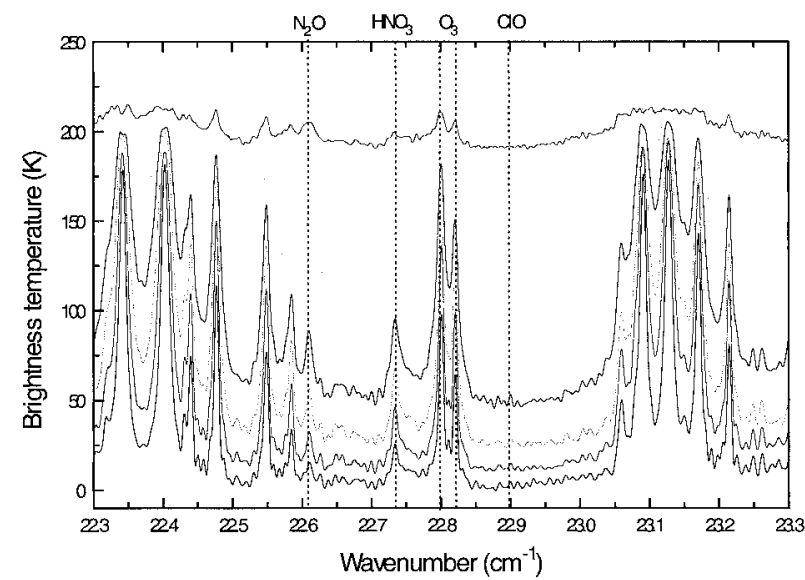

FIG. 18. Calibrated spectra of the atmospheric emission from 20$\mathrm{km}$ altitude at different limb angles. Going from the bottom curve to the top curve the spectra have the following limb angles: +10 $+3,0.0,-1,-2$, and -3 . Most of the observed features are due to ozone rotational transitions. The positions of features for some relevant atmospheric constituents are noted.

\section{g. Detector nonlinearity}

We have used the inherent rapid scanning mode to look for detector nonlinearity. If the detection system is nonlinear then the quadratic component of the nonlinear response causes an output at twice the audio frequency. Close inspection of the spectra near $46 \mathrm{~cm}^{-1}$ shows no evidence of any spectral structure above the noise level. We therefore conclude that the detector is linear for the range of signals experienced in this experiment.

\section{h. Results}

Figure 18 shows a limb-scan sequence of calibrated single spectra. The full sequence is acquired in $3 \mathrm{~min}$. We note the high transparency of the atmosphere at high limb angles and the increasing emission at progressively lower limb angles. The water vapor continuum is responsible for the rapid rise in emission in the "window" regions.

In this spectral region most of the observed atmospheric emission lines originate from ozone, along with significant emission from $\mathrm{N}_{2} \mathrm{O}$ and $\mathrm{HNO}_{3}$ and a trace amount of $\mathrm{ClO}$; line positions are marked on the figure. A clear identification of $\mathrm{ClO}$ will only be possible from a careful analysis of the spectral structure along with selective averaging of grouped spectra.

\section{Conclusions}

The SAFIRE-A instrument was successfully integrated with the M-55 Geophysica aircraft. The only problem encountered during the Arctic campaign made from Rovaniemi in winter 1996-97 was that of high acoustic noise, which prevented the use of the photo- lithographic beamsplitter and the exploitation of the second high-frequency detector channel.

A novel interferometer drive mechanism with folded optics provided useful optical compensations along with reduced linear dimensions, which made a relatively compact instrument package for a spectrometer with inherently high spectral resolution and large throughput possible.

The instrument also uses a new cold pupil and field stop optical configuration, which allows the full exploitation of narrowband filters for the reduction of photon noise. These features along with the use of stateof-the-art detectors provided unprecedented radiometric performances in the submillimeter region. At about 20 $\mathrm{cm}^{-1}$ with a 30-s measurement time the atmospheric emission spectrum is observed with an rms noise of 0.5 $\mathrm{K}$ (measured in brightness temperature) in each spectral element. A comparison of the measured $\mathrm{S} / \mathrm{N}$ with model calculations clearly shows that during in-flight operations the SAFIRE-A instrument performed close to expectation. With some refinements and continued efforts, we would expect further improvements to be forthcoming. Indeed the incorporation of yet narrower bandpass filters would give an immediate sensitivity advantage for targeted species.

This instrument makes possible the accurate observation of several key atmospheric species in the same air packet and with good time resolution along the aircraft flight path.

Acknowledgments. The development of SAFIRE-A instrument was based on the support received in Italy from the Italian Space Agency (ASI) and on the international collaboration that was established with the support of the CEC Environmental Research Programme with both Contract EV5V-CT94-0359 "SAFIRE-A Spectroscopy of the Atmosphere using Far-Infrared Emission/Airborne," and Contract ENV4-CT95-0045 "SAFIRE-A optimization and flight qualification for APE.' Support for the test flights and the Arctic campaign in Rovaniemi and program coordination was provided by the Piano Spaziale Nazionale Ricerca in Antartide (PNRA), the CEC Environmental Research Programme with Contract ENV4-CT95-0143 “The Airborne Polar Experiment (APE): An airborne study of the microphysical and chemical structure of polar stratospheric clouds," and the European Science Foundation.

In addition the German Ozone Research Programme (BMBF Grant 01L09314) provided funding for the testing of the AHRS attitude system.

The success of the qualification tests and of the campaign was possible thanks to the valuable auxiliary support provided by Ente per le Nuove Tecnologie, l'Energia e l'Ambiente (ENEA), the Myasishchev Design Bureau (MDB), and the Italian Air Force (Aereonautica Militare Italiana). Instrument preparations and flight operations were made possible by the assistance of many dedicated persons, including Ravinder Bahtia, 
Stefano Balestri, Ubaldo Bonafè, Roberto Calzolai, Renzo Regini, Giovanni Rinchiusi, Orazio Sottile, Daniele Tirelli, Massimo Trambusti, Giancarlo Valmori, Alberto Vatteroni, and Lionello Zuccagnoli.

\section{REFERENCES}

Barbis, A., R. Bonsignori, M. Brotini, V. Spicci, and B. Carli, 1994: High precision mirror drive for far-infrared high resolution Fourier spectrometer. Proc. Int. Symp. on Space Optics, Vol. 2209, Garmish, Germany, SPIE, 24-35.

—, B. Carli, U. Cortesi, and A. Gignoli, 1998: Optical path difference measurement for high resolution Fourier spectrometer. J. Opt., 29, 141-145.

Brotini, M., A. Barbis, B. Carli, F. Fabrizzi, and V. Spicci, 1993: High precision double four bar linkage mechanism for linear interferometric scanning. Proc. Fifth European Space Mechanisms Symp., ESA-SP 334, Nordwjik, Netherlands, ESTEC, 179-184.

Carli, B., 1989: High-resolution far-infrared FT spectroscopy of the stratosphere: Optimization of the optical design of the instrument. Proc. Seventh Int. Conf. on Fourier Transform Spectroscopy, Fairfax, VA. SPIE, 93-98.

_ , and M. Carlotti, 1992: Far-infrared microwave spectroscopy of the Earth's atmosphere. Spectroscopy of the Earth's Atmosphere and Interstellar Medium, N. Rao and A. Weber, Eds., Academic Press, 1-95.

- F. Mencaraglia, and A. Bonetti, 1984: Submillimeter high resolution FT spectrometer for atmospheric studies. Appl. Opt., 23, 2595-2603.
Decker, B. L., 1984: World Geodetic System 1984. Proc. Fourth Int. Geodetic Symp. on Satellite Positioning, Austin, Texas.

DeValk, J. P. J. M. M., and Coauthors, 1997: Airborne heterodyne measurements of stratospheric $\mathrm{ClO}, \mathrm{HCl}, \mathrm{O}_{3}$ and $\mathrm{N}_{2} \mathrm{O}$ during SESAME 1 over northern Europe. J. Geophys. Res., 102(D1), 1391-1398.

Holland, W. S., and Coauthors, 1996: $100 \mathrm{mK}$ bolometers for the Submillimetre Common-User Bolometer Array (SCUBA), I. Design and Construction. Proc. Int. J. Infrared and Millimetre Waves, 17, 669-692.

Kramer, H. J., 1994: Observation of the Earth and its environment. Survey of Missions and Sensors, Springer-Verlag, 317-563.

Martin, D. H., and E. Puplett, 1969: Polarised interferometric spectrometry for the millimetre and submillimetre spectrum. Infrared Phys., 10, 105-109.

Maucher, G., 1995: The pointing and the star reference system of the MIPAS-B2 Gondola, Part 2. Proc. 12th ESA Symp. on Rocket and Balloon Programmes and Related Research, Lillehammer, Norway, ESA, 505-510.

Pickett, H. M., and D. B. Peterson, 1996: Comparison of measured stratospheric $\mathrm{OH}$ with prediction. J. Geophys. Res., 101(D11), $16789-16796$.

Piesch, C., 1996: Design of an MIPAS instrument for high altitude aircraft. Proc. Second Int. Airborne Remote Sensing Conf. and Exhibition, Vol. II, San Francisco, CA, Environmental Research Institute of Michigan/NASA, 199-208.

Proffitt, M. H., and Coauthors, 1993: Ozone loss inside the northern polar vortex during the 1991-1992 winter. Science, 261, 11501154.

Seefeldner, M., and C. Keim, 1995: The pointing and the star reference system of the MIPAS-B2 Gondola, Part 1. Proc. 12th ESA Symp. on Rocket and Balloon Programmes and Related Research, Lillehammer, Norway, ESA, 505-510. 